\title{
Differential blood pressure response to continuous positive airway pressure treatment according to the circadian pattern in hypertensive patients with obstructive sleep apnoea
}

\author{
Esther Sapiña-Beltrán ${ }^{1,2}$, Gerard Torres ${ }^{1}$, Iván Benítez ${ }^{1,2}$, \\ Fernando Santamaría-Martos (10 1, Joaquín Durán-Cantolla ${ }^{2,3}$, Carlos Egea ${ }^{2,4}$, \\ Manuel Sánchez-de-la-Torre ${ }^{1,2}$, Ferrán Barbé ${ }^{1,2}$ and Mireia Dalmases ${ }^{1,2}$ \\ on behalf of the Spanish Sleep and Breathing Group ${ }^{5}$
}

@ERSpublications

Only non-dipper patients benefited from CPAP treatment in terms of BP reduction. Performing ABPM before prescribing CPAP to hypertensive patients may be important in clinical practice to predict BP response http://bit.ly/2Y1umEP

Cite this article as: Sapiña-Beltrán E, Torres G, Benítez I, et al. Differential blood pressure response to continuous positive airway pressure treatment according to the circadian pattern in hypertensive patients with obstructive sleep apnoea. Eur Respir J 2019; 54: 1900098 [https://doi.org/10.1183/13993003.000982019].

ABSTRACT Continuous positive airway pressure (CPAP) has a heterogeneous effect on blood pressure (BP) in hypertensive patients. However, the effect of CPAP on BP in hypertensive subjects regarding circadian BP pattern has never been explored. This study aimed to assess the effect of CPAP on BP, taking into consideration the circadian BP pattern in untreated hypertensive patients.

This study is a post hoc analysis of the Spanish Cohort for the Study of the Effect of CPAP in Hypertension (CEPECTA), a multicentre, randomised trial of CPAP versus sham-CPAP in patients with new-onset systemic hypertension and an apnoea-hypopnoea index $>15$ events $\cdot \mathrm{h}^{-1}$. We included patients for whom 24-h ambulatory BP monitoring (ABPM) data were available at baseline and 12 weeks after the intervention. Subjects were classified based on the dipping ratio (dipper/non-dipper). We evaluated the effect of CPAP on ABPM parameters after 12 weeks of treatment.

Overall, 272 hypertensive subjects were included in the analysis (113 dippers and 159 non-dippers). Baseline clinical and polysomnographic variables were similar between the groups. CPAP treatment in non-dipper patients was associated with reductions in 24-h ambulatory BP variables and night-time ambulatory BP measurements. However, a nonsignificant effect was reported in the dipper group. The differential effects of CPAP between the groups were $-2.99 \mathrm{mmHg}(95 \% \mathrm{CI}-5.92--0.06 \mathrm{mmHg})$ for the mean 24-h ambulatory BP and $-5.35 \mathrm{mmHg}(95 \% \mathrm{CI}-9.01--1.69 \mathrm{mmHg}$ ) for the mean night-time ambulatory BP.

Our results show a differential effect of CPAP treatment on BP in hypertensive patients depending on the circadian pattern. Only non-dipper patients benefited from CPAP treatment in terms of BP reduction.

This article has supplementary material available from erj.ersjournals.com

Received: Jan 142019 | Accepted after revision: April 212019

Copyright OERS 2019 


\section{Introduction}

Obstructive sleep apnoea (OSA) is a common sleep disorder characterised by partial (hypopnoea) or complete (apnoea) episodes of collapse in the upper airway during sleep. This collapse causes intermittent hypoxia, hypercapnia, negative intra-thoracic pressure and arousals, resulting in excessive daytime sleepiness and poor quality of life. OSA is associated with a higher cardiovascular risk due to several pathogenic factors, and hypertension has been established as one of the main causes of these cardiovascular complications [1-4]. Moreover, OSA can reportedly affect the circadian blood pressure (BP) pattern; within the OSA population there is an increased prevalence of patients with a non-dipper circadian BP pattern, which has been related to a poor cardiovascular prognosis [5-7].

Several randomised clinical trials and meta-analyses have indicated that continuous positive airway pressure (CPAP) can produce a modest but consistent reduction in $\mathrm{BP}$, with greater reductions observed in patients with resistant hypertension $(\mathrm{RH})$ [8-15]. Severe OSA, hypersomnolence, higher BP values and adherence to CPAP are variables that have been associated with a greater improvement in BP in several studies $[8,12,15]$. Nevertheless, even in $\mathrm{RH}$ patients with moderate to severe OSA and good compliance, the effect of CPAP treatment on BP is highly variable [8].

Recently, our group published a study on office normotensive patients who showed a differential BP response to CPAP depending on the dipping ratio (DR) category. The results of this study indicated that several factors may contribute to modulating the effect of CPAP on BP [16] and highlight the importance of better characterising OSA patients in terms of the BP response to CPAP treatment.

Therefore, owing to the high variability of the results observed, a precision medicine approach is necessary to identify subgroups of patients in whom BP could be reduced to a greater extent and who could benefit from CPAP treatment, and differentiate these subgroups from those who experience low or no effects of CPAP on BP [8, 17]. Determining which patients could benefit from this intervention could have important implications in clinical practice and for resource consumption.

The effects of CPAP treatment on BP in hypertensive subjects with respect to the circadian BP pattern have never been explored [18]. Thus, as previous results suggest, we hypothesise that the BP response in hypertensive patients could vary depending on the DR category. The aim of the present study was to assess the effect of CPAP treatment on BP considering the baseline circadian BP pattern in untreated hypertensive patients.

\section{Materials and methods}

\section{Design and study population}

This study is a post hoc analysis of the Spanish Cohort for the Study of the Effect of CPAP in Hypertension (CEPECTA). This was a multicentre, randomised, prospective, double-blind and parallel study controlled by a placebo that evaluated the effect of CPAP treatment on BP in patients with OSA and untreated systemic hypertension diagnosed based on office BP measurements [19].

Briefly, patients aged between 18 and 75 years with untreated new-onset systemic hypertension were included between December 2004 and June 2007. Patients were excluded if they met any of the following criteria: had secondary hypertension, had a BP $>180 / 100 \mathrm{mmHg}$, had cognitive impairment, were professional drivers or handled dangerous machinery, were shift workers, were pregnant, had severe chronic disease or were previously treated with CPAP, or had any contraindication for prescribing CPAP. Patients treated with antihypertensive, psychotropic, stimulant or antidepressant drugs and those consuming illicit drugs were also excluded.

All patients underwent polysomnography and 24-h ambulatory blood pressure monitoring (ABPM) (model 90207; Spacelabs Healthcare, Snoqualmie, WA, USA) at baseline and after 6 and 12 weeks of treatment. Subjects with an apnoea-hypopnoea index (AHI) $>15$ events $\cdot \mathrm{h}^{-1}$ were randomised to CPAP treatment or sham-CPAP (Sham) and evaluated at 12 weeks.

Affiliations: ${ }^{1}$ Hospital Universitari Arnau de Vilanova and Santa Maria, Group of Translational Research in Respiratory Medicine, IRB Lleida, Universitat de Lleida, Lleida, Spain. ${ }^{2}$ Centro de Investigación Biomédica en Red de Enfermedades Respiratorias (CIBERES), Madrid, Spain. ${ }^{3}$ Bioaraba Research Institute, OSI Araba University Hospital, Dept of Medicine of Basque Country University, Vitoria-Gasteiz, Spain. ${ }^{4}$ Unidad Funcional de Sueño, Bioaraba Research Institute, OSI Araba University Hospital, Dept of Medicine of Basque Country University, Vitoria-Gasteiz, Spain. ${ }^{5} \mathrm{~A}$ list of members of the Spanish Sleep and Breathing Group can be found in the acknowledgements section.

Correspondence: Mireia Dalmases, Hospital Universitari Arnau de Vilanova and Santa Maria, Group of Translational Research in Respiratory Medicine, IRB Lleida, Universitat de Lleida, Avda. Rovira Roure, 8025198 Lleida, Spain. E-mail: mdalmases.lleida.ics@agencat.cat 
Systemic hypertension was not treated with drugs during the study. More detailed information on the inclusion and exclusion criteria and the methodology of the CEPECTA study is published elsewhere [19].

The CEPECTA trial was conducted in accordance with the Declaration of Helsinki, and the protocol was approved by the ethics committee. All participants provided written informed consent. The trial is registered with ClinicalTrials.gov (NCT00202527).

For the present study, we selected 272 patients who completed the CEPECTA trial and for whom 24-h ABPM data were available at baseline and after 12 weeks. The included patients were classified as dippers or non-dippers based on the DR (figure 1).

\section{Procedures}

Definition of hypertension

Systemic hypertension was diagnosed according to standard criteria and defined as an office systolic blood pressure $(\mathrm{SBP}) \geqslant 140 \mathrm{mmHg}$, a diastolic blood pressure (DBP) $\geqslant 90 \mathrm{mmHg}$ or both.

\section{Sleep evaluation}

All included subjects underwent polysomnography according to recommended guidelines. The sleep evaluation was considered valid if it lasted for $>180 \mathrm{~min}$ of total sleep.

\section{4-h ABPM}

24-h ABPM (model 90207, Spacelabs Healthcare) was performed for all patients at baseline and after 6 and 12 weeks of treatment.

During ABPM, BP measurements were recorded every $20 \mathrm{~min}$ during the daytime (from 06:00 $\mathrm{h}$ to 22:00 h) and every $30 \mathrm{~min}$ during the night-time (from 22:00 h to 06:00 h).

The diagnosis of true systemic hypertension was determined based on 24-h ABPM results following guidelines and standard criteria. The cut-off for systemic hypertension was defined as an SBP $\geqslant 135 \mathrm{mmHg}, \mathrm{DBP} \geqslant 85 \mathrm{mmHg}$ or both during the awake period [19].

Circadian patterns were defined based on the DR (night-time/daytime BP ratio): non-dipper $>0.9$ and dipper $\leqslant 0.9$.

The mean $\mathrm{BP}$ was calculated using the formula $(\mathrm{SBP}+2 \times \mathrm{DBP}) / 3$.

\section{Office BP measurements}

Office BP measurements were performed following guidelines. Before BP measurement, the patient would rest for at least $5 \mathrm{~min}$ in a seated position with feet on the floor and the arm supported at the heart level. Caffeine, exercise and smoking were avoided for at least $30 \mathrm{~min}$ prior to measurement. The seated $\mathrm{BP}$ was recorded as the average of at least two replicate measurements [19, 20].

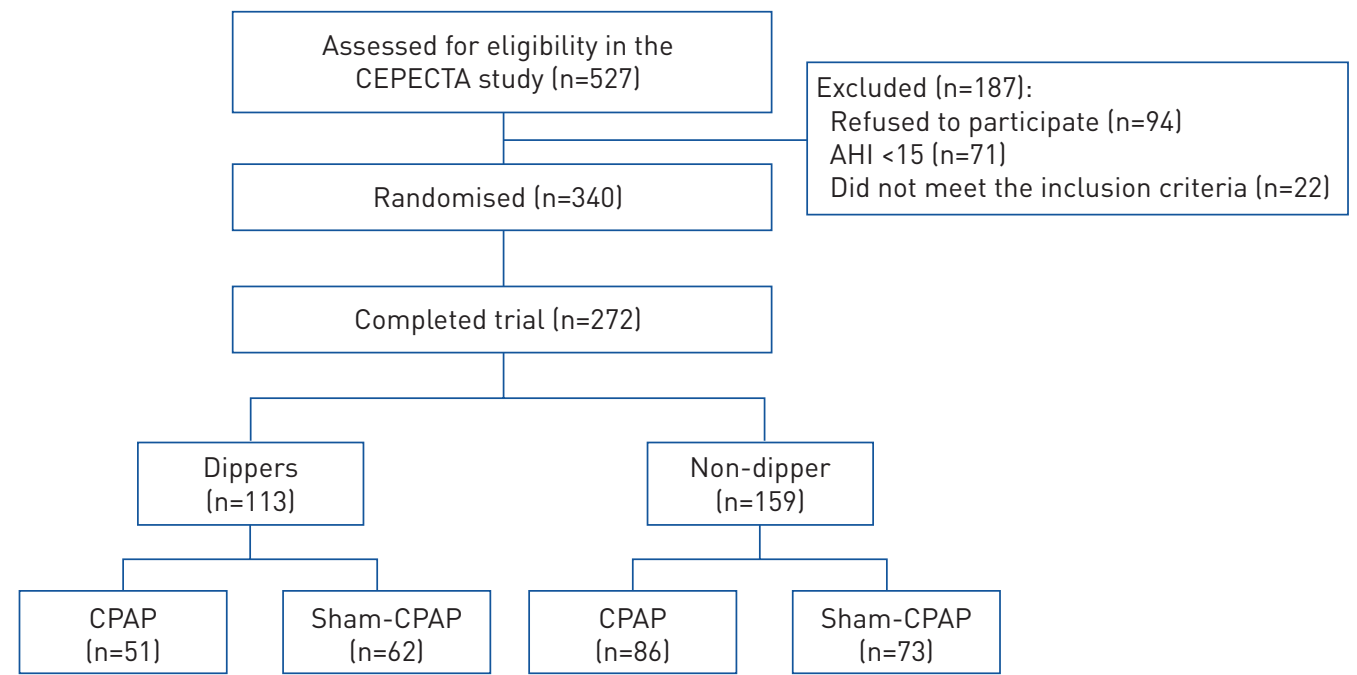

FIGURE 1 Flow diagram of the study. CEPECTA: Spanish Cohort for the Study of the Effect of CPAP in Hypertension; AHI: apnoea-hypopnoea index; CPAP: continuous positive airway pressure. 
CPAP treatment

Patients with CPAP treatment were titrated with an auto-CPAP (Autoset-T; ResMed, Sydney, Australia) according to a previously described protocol [19]. The optimal pressure was determined visually from the raw data, and the patient was treated with this pressure for 12 weeks. Furthermore, the patients receiving Sham also received the treatment at home for 12 weeks.

\section{Statistical analysis}

Baseline bivariate analysis was carried out using a t-test (or an equivalent nonparametric test) or Chi-squared test depending on whether the variables were quantitative or categorical, respectively. The differential CPAP effect on BP according to the circadian pattern was assessed using linear models. The models included the treatment (CPAP or Sham), circadian pattern (dipper or non-dipper) and the interaction between them. All models were adjusted according to baseline measurements. Furthermore, the CPAP effect was evaluated using a linear model adjusted for age, sex, body mass index (BMI) and AHI. The assumptions of the model were assessed using residual analysis. All tests were two tailed, and p-values $<0.05$ were considered statistically significant.

\section{Results}

\section{Patient characteristics}

A total of 272 hypertensive subjects with untreated hypertension and an AHI $>15$ events.h ${ }^{-1}$ were included in the analysis. Of all the patients included, 113 (42\%) were dippers, and 159 (58\%) were non-dippers.

The baseline characteristics of the enrolled subjects are shown in table 1. No significant differences were observed in the baseline variables between the groups. Mainly, the subjects included were male with a

\section{TABLE 1 Baseline characteristics of the studied groups}

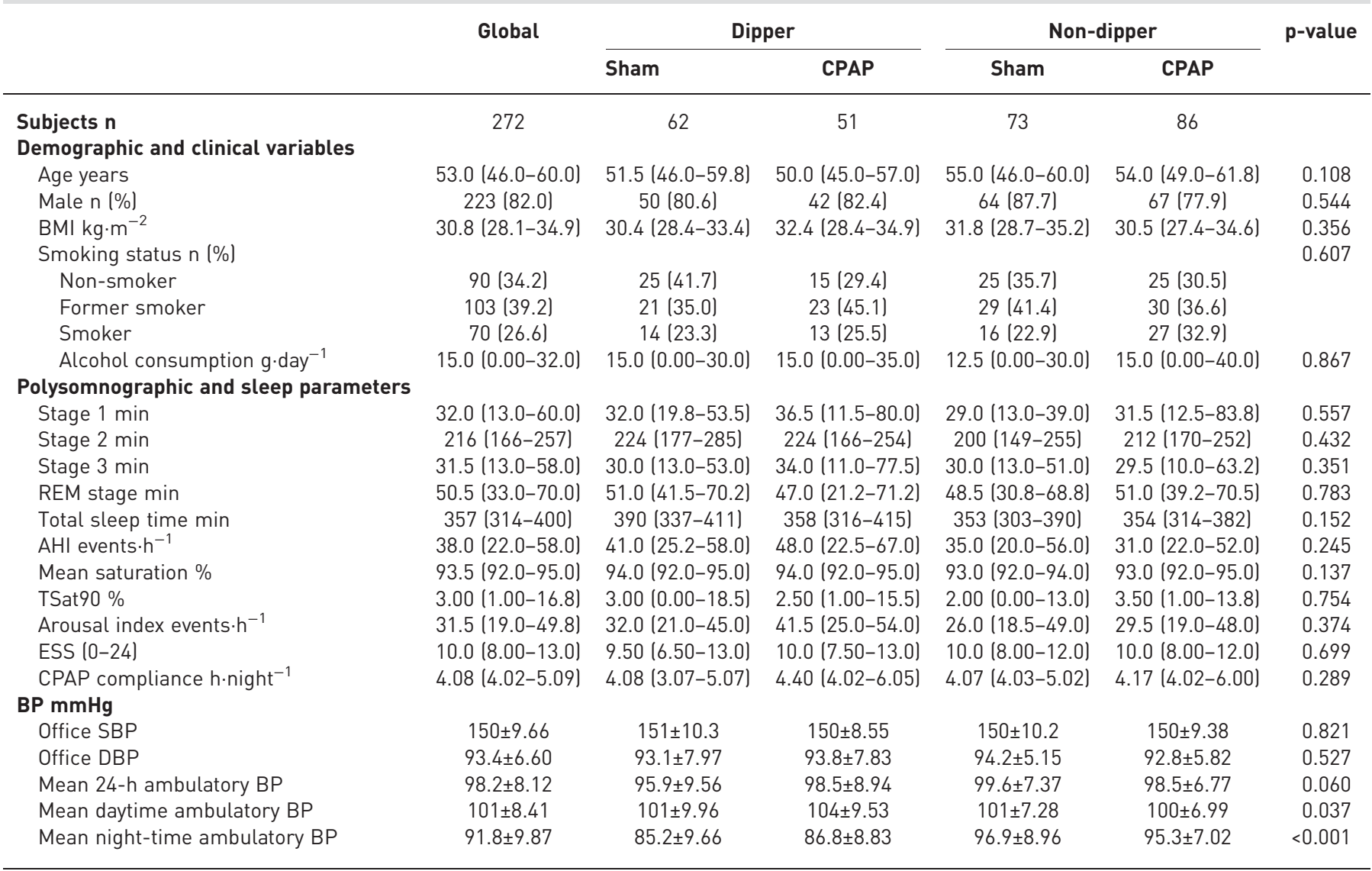

Data are presented as median (interquartile range) or mean \pm SD, unless otherwise stated. CPAP: continuous positive airway pressure; BMI: body mass index; REM: rapid eye movement; AHI: apnoea-hypopnoea index; TSat90: night-time with oxygen saturation less than 90\%; ESS: Epworth Sleepiness Scale; BP: blood pressure; SBP: systolic blood pressure; DBP: diastolic blood pressure. 
median (interquartile range) age of 53.0 years (46.0-60.0 years), BMI of $30.8 \mathrm{~kg} \cdot \mathrm{m}^{-2}\left(28.1-34.98 \mathrm{~kg} \cdot \mathrm{m}^{-2}\right.$ ) and AHI of 38.0 events. $\mathrm{h}^{-1}\left(22.0-58.0\right.$ events $\left.\cdot \mathrm{h}^{-1}\right)$. No differences were observed in polysomnographic and sleep variables between the groups. The mean \pm SD office SBP and DBP of the subjects were 150 $\pm 9.66 \mathrm{mmHg}$ and $93.4 \pm 6.60 \mathrm{mmHg}$, respectively.

\section{Effect of CPAP treatment on BP by circadian pattern}

The mean CPAP compliance was $4.08 \mathrm{~h}$, and there were no significant differences across the different groups. Changes in ABPM parameters after 12 weeks of treatment (CPAP or Sham) were evaluated in each circadian pattern group (dipper or non-dipper). In the dipper group, we observed nonsignificant effects of CPAP on the mean 24-h ambulatory BP $(0.11 \mathrm{mmHg}, 95 \% \mathrm{CI}-2.13-2.34 \mathrm{mmHg})$, the mean daytime ambulatory $\mathrm{BP}(-0.21 \mathrm{mmHg}, 95 \% \mathrm{CI}-2.65-2.23 \mathrm{mmHg})$ and the mean night-time ambulatory BP $(0.95 \mathrm{mmHg}, 95 \% \mathrm{CI}-1.84-3.73 \mathrm{mmHg})$. In contrast, significant effects of CPAP were observed on the mean 24 -h ambulatory $\mathrm{BP}(-2.88 \mathrm{mmHg}, 95 \% \mathrm{CI}-4.74--1.02 \mathrm{mmHg})$ and the mean night-time ambulatory BP $(-4.40 \mathrm{mmHg}, 95 \% \mathrm{CI}-6.73--2.08 \mathrm{mmHg})$ in the non-dipper group. The differential effects of CPAP between the circadian pattern groups revealed a better response in non-dippers of $-2.99 \mathrm{mmHg}(95 \% \mathrm{CI}-5.92--0.06 \mathrm{mmHg})$ for the mean $24-\mathrm{h}$ ambulatory BP and $-5.35 \mathrm{mmHg}(95 \%$ CI $-9.01--1.69 \mathrm{mmHg}$ ) for the mean night-time ambulatory BP (figure 2 and table 2). More detailed information on absolute BP values and changes in each treatment and dipping category group is described in supplementary table S1. The model adjusted only for baseline measurements showed similar results (supplementary table S2).

When the analysis was performed with only patients diagnosed with systemic hypertension based on the results of 24-h ABPM, similar results were observed. A differential effect of CPAP was also observed between dippers and non-dippers. Non-dipper patients exhibited significantly reduced BP, especially night-time BP (supplementary table S3), while no significant changes were observed in the dipper group (supplementary table S3).

\section{Discussion}

This study suggests that the BP response to CPAP treatment in hypertensive patients depends on the circadian BP pattern. The results show significant reductions in the mean 24-h ambulatory BP, 24-h systolic BP, 24-h diastolic BP and night-time ambulatory BP variables in non-dipper patients, whereas no significant changes were observed in the dipper group after 12 weeks of CPAP treatment. Therefore, in relation to BP, only non-dipper patients benefited from CPAP treatment.

Several studies have evaluated the effect of CPAP treatment on BP in hypertensive patients, and a reduction in BP has been described previously $[19,21,22]$. Data from a meta-analysis indicate a mild effect of CPAP treatment on BP in OSA patients, which has been reported to be $\sim 2 \mathrm{mmHg}[13-15,23$, 24], with greater reductions observed in subjects with $\mathrm{RH}$ [8-11]. Notably, the BP response to CPAP treatment is highly variable. Some authors noted that factors such as higher BP values, CPAP use or OSA severity could be related to a better BP response [10, 12, 25]; nevertheless, the causes of this variability have not been well established.

This considerable variability in the BP response to CPAP treatment highlights the importance of differentiating patients who will effectively respond to CPAP treatment from those who will not. In recent years, some published studies have identified clinical and molecular profiles that may predict the BP response to CPAP treatment. SANCHEZ-DE-LA TORRE et al. [17] reported a circulating miRNA profile that could predict BP responses to CPAP treatment in patients with RH and OSA. In an observational study of patients with severe OSA, CASTRO-GRATTONi et al. [26] suggested that the circadian BP pattern, nocturnal hypertension and the night-time heart rate could be predictors of BP response to CPAP treatment. Data from an observational study recently published by our group showed that in normotensive subjects, the BP circadian pattern may influence the BP response to CPAP treatment [16]. Although several authors have suggested that certain clinical characteristics may predict the BP response to treatment, no randomised studies have confirmed these findings, which could be very useful in clinical practice.

In our study, the decrease observed in the mean 24 -h ambulatory BP was $-2.99 \mathrm{mmHg}$. When analysing nocturnal $\mathrm{BP}$, the decrease was $-5.35 \mathrm{mmHg}$ in the mean night-time ambulatory $\mathrm{BP},-6.45 \mathrm{mmHg}$ in the mean systolic ambulatory BP and $-4.87 \mathrm{mmHg}$ in the mean diastolic ambulatory BP.

In previous studies of systemic hypertension and $\mathrm{RH}$ patients, decreases in the mean 24-h ambulatory BP of approximately $-1.5 \mathrm{mmHg}$ [19] and $-3 \mathrm{mmHg}$, respectively, were observed [8,9]. Our results show that $\mathrm{BP}$ decreases are greater in non-dipper patients than those in dipper patients, and non-dipper patients would therefore benefit the most from CPAP treatment in terms of BP reduction. 

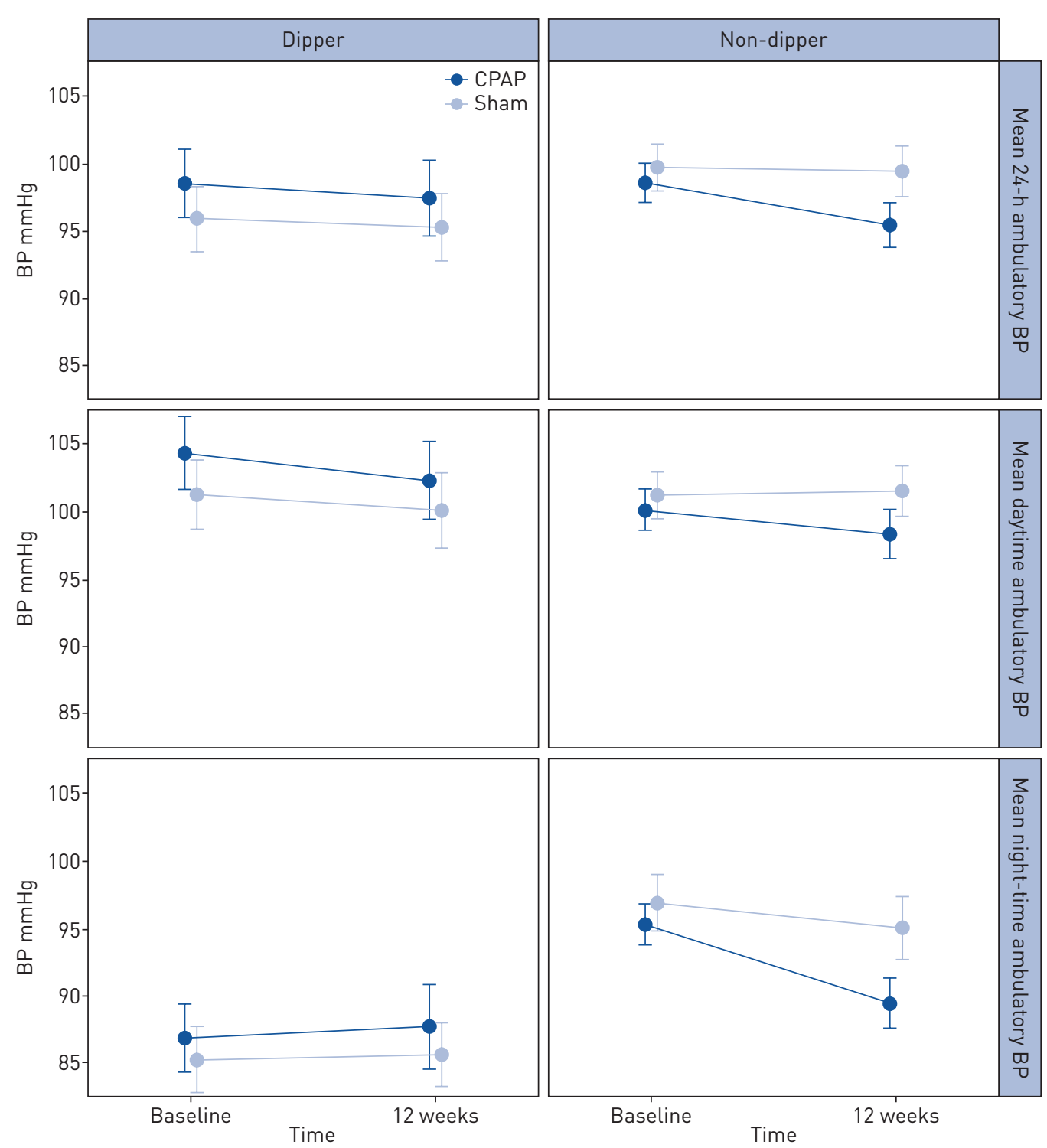

FIGURE 2 Changes in blood pressure (BP) parameters at 12 weeks according to the circadian pattern for the dipper group and the non-dipper group. The figure shows the means $(95 \%$ confidence intervals) of the observed values for 24-h ambulatory BP, daytime BP and night-time BP at baseline and after 12 weeks of treatment according to the circadian pattern. In the non-dipper group, the figure shows a greater reduction in $\mathrm{BP}$ in patients with continuous positive airway pressure (CPAP) compared with those receiving sham-CPAP treatment. In the dipper group, no differential effect was observed.

Multiple studies have indicated that sleep alterations, such as duration changes, fragmentation or blunted BP declines, which are frequent in OSA, are associated with atherosclerosis, hypertension and an increased incidence of cardiovascular events $[27,28]$.

Previous studies have highlighted the importance of reducing night-time BP and have suggested that decreasing nocturnal BP has higher prognostic value for reducing cardiovascular outcomes than reducing daytime BP $[29,30]$. The fact that these reductions in BP are observed in non-dipper subjects is especially important because this circadian pattern category has been related to a worse cardiovascular prognosis, a higher prevalence of organ damage and less favourable outcomes compared to the dipper category [31, 32]. International guidelines indicate that even minimal reductions in BP levels may be clinically effective in reducing cardiovascular mortality [20].

Therefore, identifying bedtime chronotherapy strategies to reduce BP in hypertensive patients, especially at night, has received increasing interest [33], and some authors have even combined these strategies with CPAP treatment in hypertensive patients with OSA [34]. However, the results are not fully consistent, and more evidence is required to confirm the beneficial effect of combining both methods. 
TABLE 2 Changes in blood pressure by circadian pattern groups adjusted by confounding factors

\begin{tabular}{|c|c|c|c|c|c|c|}
\hline Blood pressure & \multicolumn{2}{|l|}{ Dipper $\#$} & \multicolumn{2}{|c|}{ Non-dipper ${ }^{\Uparrow}$} & \multicolumn{2}{|l|}{ Difference } \\
\hline \multicolumn{7}{|c|}{ 24-h ABPM mmHg } \\
\hline Mean & $0.11(-2.13-2.34)$ & 0.9997 & $-2.88(-4.74--1.02)$ & 0.0139 & $-2.99(-5.92--0.06)$ & 0.0458 \\
\hline Systolic & $-0.11(-3.22-2.99)$ & 0.9999 & $-3.63(-6.21--1.04)$ & 0.032 & $-3.52(-7.58-0.55)$ & 0.0898 \\
\hline Diastolic & $0.16(-1.92-2.25)$ & 0.9987 & $-2.49(-4.23--0.76)$ & 0.0267 & $-2.66(-5.4-0.08)$ & 0.0573 \\
\hline \multicolumn{7}{|c|}{ Daytime ABPM mmHg } \\
\hline Diastolic & $-0.24(-2.56-2.09)$ & 0.9971 & $-2.01(-3.93--0.08)$ & 0.1762 & $-1.77(-4.81-1.28)$ & 0.2538 \\
\hline \multicolumn{7}{|c|}{ Night-time ABPM mmHg } \\
\hline Mean & $0.95(-1.84-3.73)$ & 0.9097 & $-4.4(-6.73--2.08)$ & 0.0014 & $-5.35(-9.01--1.69)$ & 0.0043 \\
\hline Systolic & $0.48(-3.5-4.45)$ & 0.9955 & $-5.97(-9.29--2.65)$ & 0.0028 & $-6.45(-11.66--1.23)$ & 0.0155 \\
\hline Diastolic & $1.21(-1.33-3.74)$ & 0.788 & $-3.66(-5.78--1.54)$ & 0.0046 & $-4.87(-8.2--1.53)$ & 0.0044 \\
\hline
\end{tabular}

This study shows a differential BP response based on the circadian pattern category in hypertensive patients and demonstrated the importance of performing ABPM in hypertensive subjects before prescribing CPAP. The results support the current recommendation of performing ABPM for the management of patients with office hypertension and suspected OSA. However, this recommendation was based on an assessment of cardiovascular risk [35, 36], whereas our study was based on identifying patients who could most benefit from CPAP treatment in terms of BP. Our study indicates that the BP circadian pattern at baseline may determine differences in the BP response to CPAP treatment. Therefore, performing ABPM may help to identify subgroups of hypertensive patients with OSA in whom a greater reduction of BP is expected. Our results highlight the utility of individual characteristics, such as the BP circadian pattern, as factors to consider in the management of these patients.

Our results suggest that the BP circadian pattern can be used when selecting the optimal therapeutic approach for each patient, and that CPAP treatment may be the first treatment approach in untreated hypertensive patients with OSA who are non-dippers.

Future studies should determine the role of ABPM in managing these patients and clarify whether ABPM should be routinely performed for patients with hypertension and OSA before deciding to prescribe CPAP.

The main strength of this study is that, to our knowledge, it is the first to confirm that the effect of CPAP on $\mathrm{BP}$ varies depending on the circadian pattern and to identify the dipping category as a clinical variable that differentiates between hypertensive OSA patients with a good BP response to CPAP treatment and those without a good response. Moreover, this study included a large sample size, had ABPM implemented and used polysomnography to diagnose OSA. The included patients did not receive antihypertensive drugs and were treated only with conservative measures [19], thus enabling the effects of CPAP to be evaluated without interference from changes in BP induced by pharmacological treatments. Nevertheless, the study has several limitations. First, this study has the inherent limitations of a post hoc analysis. In our analysis, the included patients were classified according to the DR, which was not considered before randomisation. However, dippers and non-dippers were fully comparable at the baseline evaluation, and when we assessed the effect of CPAP, a fully adjusted model showed similar results. Second, the subjects presented with moderate to severe OSA, and the results may not be generalisable to patients with less severe OSA. Third, the included patients had recently been diagnosed with hypertension, exhibited mild hypertension and were untreated. Thus, the results should be interpreted with caution when addressing patients receiving antihypertensive drugs. Fourth, the categorisation of the circadian BP pattern with single 24-h ABPM is only moderately reproducible.

\section{Conclusions}

Our findings show that in patients with moderate to severe OSA who are newly diagnosed with hypertension, only those with a non-dipper BP circadian pattern experience significant benefits for $\mathrm{BP}$ with CPAP treatment, especially nocturnal BP. 
Acknowledgements: Members of the Spanish Sleep and Breathing Group are as follows: Felipe Aizpuru, Jose María Montserrat, Eugeni Ballester, Jose Ignacio Aguirregomoscorta, Mónica Gonzalez, Patricia Lloberes, Juan Fernando Masa, Mónica de La Peña, Santiago Carrizo and Mercedes Mayos.

Author contributions: E. Sapiña-Beltrán, G. Torres, J. Durán-Cantolla, C. Egea, M. Sánchez-de-la-Torre, F. Barbé and M. Dalmases participated in the study conception and design. I. Benítez and F. Santamaría-Martos participated in the analysis of the data. E. Sapiña-Beltrán, G. Torres, I. Benítez, F. Santamaría-Martos, M. Sánchez-de-la-Torre, F. Barbé and M. Dalmases participated in the interpretation of the data for the work. All authors drafted the manuscript, revised it critically and provided final approval of the version to be published. M. Dalmases is the guarantor.

Conflict of interest: None declared.

Support statement: This work is supported by Fondos recibidos por el ISCIII y fondos FEDER "Una manera de hacer Europa" (PI16/00489).

\section{References}

1 Sapina E, Torres G, Barbe F, et al. The use of precision medicine to manage obstructive sleep apnea treatment in patients with resistant hypertension: current evidence and future directions. Curr Hypertens Rep 2018; 20: 60.

2 Pepin JL, Borel AL, Tamisier R, et al. Hypertension and sleep: overview of a tight relationship. Sleep Med Rev 2014; 18: 509-519.

3 Sánchez-de-la-Torre M, Campos-Rodriguez F, Barbé F. Obstructive sleep apnoea and cardiovascular disease. Lancet Respir Med 2013; 1: 61-72.

4 Lévy P, Kohler M, McNicholas WT, et al. Obstructive sleep apnoea syndrome. Nat Rev Dis Prim 2015; 1: 1-20.

5 Pankow W, Nabe B, Lies A, et al. Influence of obstructive sleep apnoea on circadian blood pressure profile. J Sleep Res 1995; 4: 102-106.

6 Suzuki T, Guilleminault C, Otsuka T, et al. Blood pressure "dipping" and "non-dipping" obstructive sleep apnea syndrome patients. Sleep 2018; 19: 382-387.

7 Marrone O, Bonsignore MR. Blood-pressure variability in patients with obstructive sleep apnea: current perspectives. Nat Sci Sleep 2018; 10: 229-242.

8 Martínez-García M-A, Capote F, Campos-Rodríguez F, et al. Effect of CPAP on blood pressure in patients with obstructive sleep apnea and resistant hypertension: the HIPARCO randomized clinical trial. JAMA 2013; 310: 2407-2415.

9 Pedrosa RP, Drager LF, De Paula LKG, et al. Effects of OSA treatment on BP in patients with resistant hypertension: a randomized trial. Chest 2013; 144: 1487-1494.

10 Lozano L, Tovar JL, Sampol G, et al. Continuous positive airway pressure treatment in sleep apnea patients with resistant hypertension: a randomized, controlled trial. J Hypertens 2010; 28: 2161-2168.

11 Muxfeldt E, Margallo V, Costa LMS, et al. Effects of continuous positive airway pressure treatment on clinic and ambulatory blood pressures in patients with obstructive sleep apnea and resistant hypertension. Hypertension 2015; 65: 736-742.

12 Fava C, Dorigoni S, Dalle Vedove F, et al. Effect of CPAP on blood pressure in patients with OSA/hypopnea: a systematic review and meta-analysis. Chest 2014; 145: 762-771.

13 Iftikhar IH, Valentine CW, Bittencourt LRA, et al. Effects of continuous positive airway pressure on blood pressure in patients with resistant hypertension and obstructive sleep apnea: a meta-analysis. J Hypertens 2014; 32: 2341-2350.

14 Liu L, Cao Q, Guo Z, et al. Continuous positive airway pressure in patients with obstructive sleep apnea and resistant hypertension: a meta-analysis of randomized controlled trials. J Clin Hypertens 2016; 18: 153-158.

15 Montesi SB, Edwards BA, Malhotra A, et al. The effect of continuous positive airway pressure treatment on blood pressure: a systematic review and meta-analysis of randomized controlled trials. J Clin Sleep Med 2012; 8: 587-596.

16 Sapiña-Beltrán E, Santamaria-Martos F, Benítez I, et al. Normotensive patients with obstructive sleep apnoea: changes in 24-h ambulatory blood pressure monitoring with continuous positive airway pressure treatment. J Hypertens 2019; 37: 720-727.

17 Sánchez-De-La-Torre M, Khalyfa A, Sánchez-De-La-Torre A, et al. Precision medicine in patients with resistant hypertension and obstructive sleep apnea blood pressure response to continuous positive airway pressure treatment. J Am Coll Cardiol 2015; 66: 1023-1032.

18 Akashiba T, Minemura H, Yamamoto H, et al. Nasal continuous positive airway pressure changes blood pressure "non-dippers" to "dippers" in patients with obstructive sleep apnea. Sleep 1999; 22: 849-853.

19 Duran-Cantolla J, Aizpuru F, Montserrat JM, et al. Continuous positive airway pressure as treatment for systemic hypertension in people with obstructive sleep apnoea: randomised controlled trial. BMJ 2010; 341: c5991.

20 Chobanian AV, Bakris GL, Black HR, et al. The seventh report of the Joint National Committee on Prevention, Detection, Evaluation, and Treatment of High Blood Pressure: the JNC 7 report. JAMA 2003; 289: 2560-2572.

21 Campos-Rodriguez F, Perez-Ronchel J, Grilo-Reina A, et al. Long-term effect of continuous positive airway pressure on bp in patients with hypertension and sleep apnea. Chest 2007; 132: 1847-1852.

22 Barbe F, Duran-Cantolla J, Capote F, et al. Long-term effect of continuous positive airway pressure in hypertensive patients with sleep apnea. Am J Respir Crit Care Med 2010; 181: 718-726.

23 Bazzano LA, Khan Z, Reynolds K, et al. Effect of nocturnal nasal continuous positive airway pressure on blood pressure in obstructive sleep apnea. Hypertension 2007; 50: 417-423.

24 Bratton DJ, Stradling JR, Barbé F, et al. Effect of CPAP on blood pressure in patients with minimally symptomatic obstructive sleep apnoea: a meta-analysis using individual patient data from four randomised controlled trials. Thorax 2014; 69: 1128-1135.

25 Wolf J, Hering D, Narkiewicz K. Non-dipping pattern of hypertension and obstructive sleep apnea syndrome. Hypertens Res 2010; 33: 867-871.

26 Castro-Grattoni AL, Torres G, Martínez-Alonso M, et al. Blood pressure response to CPAP treatment in subjects with obstructive sleep apnoea: the predictive value of 24 -h ambulatory blood pressure monitoring. Eur Respir J 2017; 50: 1700651 . 
27 Hermida RC, Ayala DE, Mojón A, et al. Decreasing sleep-time blood pressure determined by ambulatory monitoring reduces cardiovascular risk. J Am Coll Cardiol 2011; 58: 1165-1173.

28 Domínguez F, Fuster V, Fernández-Alvira JM, et al. Association of sleep duration and quality with subclinical atherosclerosis. J Am Coll Cardiol 2019; 73: 134-144.

29 Roush GC, Fagard RH, Salles GF, et al. Prognostic impact from clinic, daytime, and night-time systolic blood pressure in nine cohorts of 13,844 patients with hypertension. J Hypertens 2014; 32: 2332-2340.

30 Brien EO, Parati G, Stergiou G, et al. European Society of Hypertension position paper on ambulatory blood pressure monitoring. J Hypertens 2013; 31: 1731-1768.

31 Konecny T, Kara T, Somers VK. Obstructive sleep apnea and hypertension: an update. Hypertens 2014; 63: 203-209.

32 Williams B, Mancia G, Spiering W, et al. 2018 ESC/ESH guidelines for the management of arterial hypertension: the Task Force for the management of arterial hypertension of the European Society of Cardiology and the European Society of Hypertension. J Hypertens 2018; 36: 1953-2041.

33 Hermida RC, Ayala DE, Fernández JR, et al. Bedtime blood pressure chronotherapy significantly improves hypertension management. Heart Fail Clin 2017; 13: 759-773.

34 Serinel Y, Yee BJ, Grunstein RR, et al. Chronotherapy for hypertension in obstructive sleep apnoea (CHOSA): a randomised, double-blind, placebo-controlled crossover trial. Thorax 2017; 72: 550-558.

35 Parati G, Lombardi C, Hedner J, et al. Recommendations for the management of patients with obstructive sleep apnoea and hypertension. Eur Respir J 2013; 41: 523-538.

36 Mancia G, Fagard R, Narkiewicz K, et al. 2013 ESH/ESC guidelines for the management of arterial hypertension: The Task Force for the management of arterial hypertension of the European Society of Hypertension (ESH) and of the European Society of Cardiology (ESC). Eur Heart J 2013; 34: 2159-2219. 